\title{
Erica L. Johnson, Caribbean Ghostwriting
}

\section{Alessandro Corio}

\section{(2) OpenEdition}

\section{Journals}

\section{Edizione digitale}

URL: http://journals.openedition.org/studifrancesi/6132

DOI: 10.4000/studifrancesi.6132

ISSN: 2421-5856

\section{Editore}

Rosenberg \& Sellier

\section{Edizione cartacea}

Data di pubblicazione: 1 mai 2011

Paginazione: 225-226

ISSN: 0039-2944

\section{Notizia bibliografica digitale}

Alessandro Corio, «Erica L. Johnson, Caribbean Ghostwriting», Studi Francesi [Online], 163 (LV | I) | 2011 , online dal 30 novembre 2015, consultato il 14 janvier 2021. URL: http://journals.openedition.org/ studifrancesi/6132 ; DOI: https://doi.org/10.4000/studifrancesi.6132

Questo documento è stato generato automaticamente il 14 janvier 2021.

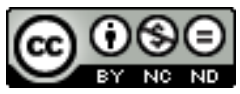

Studi Francesi è distribuita con Licenza Creative Commons Attribuzione - Non commerciale - Non opere derivate 4.0 Internazionale. 


\title{
Erica L. Johnson, Caribbean Ghostwriting
}

\author{
Alessandro Corio
}

\section{NOTIZIA}

ERICA L. JOHNSON, Caribbean Ghostwriting, Madison-Teaneck, Fairleigh Dickinson University Press, 2009, pp. 152.

1 Il saggio di Erica L. Johnson mette a fuoco una serie di problematiche fondamentali, che negli ultimi anni hanno acquisito un'importanza sempre maggiore negli studi comparatistici di orientamento postcoloniale sulle letterature caraibiche ed afroamericane. Si tratta, insomma, di una prospettiva critica che si situa all'incrocio tra gli studi femministi e postcoloniali, la storiografia subalterna, gli studi sulla diaspora afroamericana e quelli sulle narrazioni e la memoria del trauma. L'autrice individua nella ghostwriting un vero e proprio genere letterario, legato alle caratteristiche specifiche del vissuto storico dei popoli della tratta e della schiavitù: un vissuto percorso da vuoti e da silenzi, da cancellature operate dalle narrazioni storiche dominanti ed eurocentriche e, al tempo stesso, segnato dall'impossibilità di raccontare dei vissuti altamente traumatici (non è un caso che dei personaggi femminili siano al centro dei tre romanzi analizzati dall'autrice). Le tre scrittrici caraibiche sulle quali si concentra l'analisi della Johnson - Michelle Cliff, Maryse Condé e Dionne Brand - si confrontano nei loro testi letterari con le complesse problematiche della rappresentazione del «silenzio del subalterno», dei vuoti storiografici e della narrazione, possibile o impossibile, di un passato collettivo disperso e frammentato. Si tratta, insomma, di quella che Édouard Glissant ha definito, nel Discours antillais (1981), come «non-histoire» ovvero l'impossibilità della costruzione simbolica di una narrazione storica lineare e logico-sequenziale del vissuto dei popoli della tratta.

Col termine ghostwriting, l'autrice si riferisce alla scrittura letteraria di storie, eventi, vite individuali che sono state rimosse o comunque non-iscritte nella narrazione 
collettiva ed egemone; storie cancellate o messe in secondo piano dagli storici, trascurate dai cronisti, estromesse dagli archivi, insomma, vite subalterne dimenticate o rimosse dalle narrazioni storiche ufficiali e, pertanto, dalla coscienza collettiva. «The Ghost», scrive Johnson, «is by definition a witness to events from an individual's life or from a collective past that have been repressed either wilfully or unconsciously" (p. 12) e la particolare attenzione che a questa figura è stata rivolta da parte di filosofi e studiosi negli ultimi decenni è probabilmente dovuta proprio alla sua capacità simbolica di significare, al tempo stesso, l'assenza e la presenza di qualcosa («la presenza di un'assenza»). Il ruolo della scrittura nei confronti di ciò che è stato cancellato dalla Storia non si limita, dunque, ad una semplice ricostruzione dei vuoti e delle cancellature degli archivi o all'illusione di un pieno recupero della "coscienza" del soggetto subalterno. I rischi di tale ambizione sono stati ripetutamente denunciati dalla teoria postcoloniale, soprattutto da Gayatri C. Spivak la quale, nei suoi saggi sull'irrappresentabilità del soggetto subalterno, ha messo in guardia il critico e lo scrittore postcoloniale dalla tentazione-illusione di una piena ri-appropriazione del passato. Il rischio maggiore di una tale postura sarebbe quello di cancellare nuovamente il luogo del silenzio del subalterno e la «violenza epistemica» che l'ha prodotto, creando un effetto-soggetto («a subject effect») sostitutivo e falsamente autentico, che porterebbe a compimento il progetto coloniale stesso. La questione etica risulta centrale in queste contro-narrazioni e, come afferma l'autrice, «[...] the historically oppressed subject cannot be summarily represented; the conditions of archival or discursive erasure require a careful ethical stance on the part of anyone writing about these "lost" subjects» (p. 18).

Una delle caratteristiche della ghostwriting secondo Erica L. Johnson, che ne sottolinea chiaramente la differenza col romanzo storico di impianto realistico, è proprio quella di saper evitare il rischio di riprodurre la violenza epistemica enfatizzando, anche attraverso delle tecniche di narrazione postmoderna, ironica ed autocosciente, l'irrappresentabilità stessa e l'impossibilità di ricostruire la pienezza della voce e della coscienza del/della subalterno/a. Riprendendo una celeberrima affermazione di Toni Morrison (il cui romanzo-capolavoro Beloved è considerato una sorta di testo fondatore di questo genere), la ghostwriting si occuperebbe non della ricostruzione del passato, bensì della scandalosa e «contrappuntistica» (Said) esposizione narrativa dei suoi vuoti e dei suoi silenzi, di ciò che è «unspoken», ossia che è stato cancellato dalla storiografia dominante, ma anche di ciò che è «unspeakable», che non può essere raccontato né rappresentato (per questo la sua analisi si pone all'incrocio tra i subaltern studies e i trauma studies). In questo modo l'evento non-simbolizzato nel passato ritornerebbe nel presente sotto forma spettrale, stregandolo ed impedendogli così, in un certo senso, di «presentificarsi», ossia di assumere un posto fisso e stabile nella catena significante del discorso storico dominante.

E' quello che avviene, per prendere un solo esempio, nel romanzo Moi, Tituba, sorcière ... Noire de Salem (1986) di Maryse Condé, in cui la scrittrice re-inventa una vita alla schiava nera Tituba, accusata di stregoneria durante il celebre processo alle streghe di Salem nel 1692. L'operazione letteraria e l'«immaginazione etica» di Condé non si limitano, secondo la critica, a produrre un racconto realistico ed autorevole della vita di Tituba, di cui rimangono peraltro solo alcune tracce negli archivi storici del processo, ma costruisce il personaggio di Tituba come un «significante fluttuante» (riprendendo la celebre definizione di Lacan) dotandolo così di una consapevolezza postmoderna e straniante del proprio destino di negazione e di oblìo. Tale 
consapevolezza, anacronistica e carica di ironia, contribuisce a scardinare (decentrandola anche geograficamente oltre che epistemicamente) la narrazione egemone e positivista del passato e del presente, che ha costruito la figura di Tituba come «colpevole»: «Condé's Tituba breaks the signifying chain of positivist historiography in her creation of a Tituba who functions as a floating signifier. [...] It is this awareness of the concurrent projects of imagining silenced histories and narrating the present moment that informs the ethical imagination» (pp. 89 e 96).

5 Solo in questo modo, dunque, la narrazione dei silenzi e dei vuoti del passato è in grado di ritornare nel presente, di «possederlo» («to haunt») sotto forma spettrale, interrogandolo violentemente e costringendolo a rimettere in discussione le proprie modalità cognitive e le proprie forme di rappresentazione della Storia. Come scrive Toni Morrison in un celebre passaggio di Beloved, citato dall'autrice, «recognizable but indecipherable [...] were the thoughts of the women of 124 , unspeakable thoughts, unspoken» (p. 33). 